\title{
Inclusive Jets in PHP
}

\author{
Philipp Roloff*' \\ CERN \\ E-mail: philipp.roloffecern.ch
}

Differential inclusive-jet cross sections have been measured in photoproduction for boson virtualities $Q^{2}<1 \mathrm{GeV}^{2}$ with the ZEUS detector at HERA using an integrated luminosity of $300 \mathrm{pb}^{-1}$. Jets were identified in the laboratory frame using the $k_{T}$, anti- $k_{T}$ or SIScone jet algorithms. Cross sections are presented as functions of the jet pseudorapidity, $\eta^{\text {jet }}$, and the jet transverse energy, $E_{T}^{\text {jet }}$. Next-to-leading-order QCD calculations give a good description of the measurements, except for jets with low $E_{T}^{\text {jet }}$ and high $\eta^{\text {jet }}$. The cross sections have the potential to improve the determination of the PDFs in future QCD fits. Values of $\alpha_{s}\left(M_{Z}\right)$ have been extracted from the measurements based on different jet algorithms. In addition, the energy-scale dependence of the strong coupling was determined.

The European Physical Society Conference on High Energy Physics

18-24 July 2013

Stockholm, Sweden

\footnotetext{
* Speaker.

${ }^{\dagger}$ On behalf of the ZEUS collaboration.
} 


\section{Introduction}

The measurement of jet photoproduction (PHP) at HERA provides a high-statistics test of perturbative QCD (pQCD) in a process with a single hard scale, $E_{T}^{\text {jet }}$. Jet cross sections allow precise determinations of the strong coupling constant, $\alpha_{s}$, and its energy dependence.

At leading order, so-called direct and resolved processes contribute to jet photoproduction. In direct processes, the photon interacts directly with a parton in the proton. On the other hand, the photon acts as a source of partons for the resolved contributions. Hence inclusive-jet cross sections are directly sensitive to the proton and photon PDFs.

The $k_{T}$ cluster algorithm [1] in the longitudinally invariant inclusive mode [2] results in small theoretical uncertainties and hadronisation corrections in electron-proton collisions. It yields infrared- and collinear-safe cross sections at any order of QCD. More recently, new infrared- and collinear-safe algorithms like anti- $k_{T}$ [3] or SIScone [4] were developed. Jet photoproduction at HERA represents a well-understood hadron-induced reaction to test and compare the performances of these different jet clustering algorithms.

NLO QCD predictions [5] are compared to the measured inclusive-jet cross sections. The renormalisation and factorisation scales were set to $\mu_{R}=\mu_{F}=E_{T}^{\mathrm{jet}}$ and the number of flavours was chosen to be five. Unless explicitly stated otherwise, the ZEUS-S [6] parametrisations were used for the proton PDFs and the GRV-HO [7] sets were chosen for the photon PDFs. Hadronisation corrections were obtained using the Pythia [8] and Herwig [9] Monte Carlo (MC) programs. For comparisons, samples of Pythia including multi-parton interactions [10], Pythia-MI, were used to estimate the contribution from non-perturbative effects not related to hadronisation. For all three jet algorithms introduced above, missing terms beyond NLO represent the dominant uncertainty of the predictions.

\section{Differential inclusive-jet cross sections}

Single- and double-differential inclusive-jet cross sections have been measured in the reaction $e p \rightarrow e+$ jet $+\mathrm{X}$ for $142<W_{\gamma p}<293 \mathrm{GeV}$, where $W_{\gamma p}$ is the $\gamma p$ centre-of-mass energy, and $Q^{2}<1 \mathrm{GeV}^{2}$ with the ZEUS detector at HERA using an integrated luminosity of $300 \mathrm{pb}^{-1}$. The cross sections include every jet with $E_{T}^{\text {jet }}>17 \mathrm{GeV}$ and $-1<\eta^{\text {jet }}<2.5$ [11].

Single-differential cross sections based on the $k_{T}$ algorithm as functions of $E_{T}^{\text {jet }}$ and $\eta^{\text {jet }}$ are shown in Fig. 1 . The uncertainty on the jet energy scale of $\pm 1 \%$ typically leads to a $\mp 5 \%$ uncertainty on the measured cross sections which is fully correlated between measurements in different bins. At high $E_{T}^{\text {jet }}$ this uncertainty increases to $\mp 10 \%$. The measurements are well described by NLO QCD except for $\eta^{\text {jet }}>2$. The disagreement in the forward region disappears if the kinematic region of the measurement is restricted to $E_{T}^{\text {jet }}>21 \mathrm{GeV}$.

Alternative NLO QCD predictions based on the HERAPDF1.5 [12] and MSTW08 [13] proton PDFs instead of ZEUS-S are also shown in Fig. 1. The predictions based on HERAPDF1.5 are lower than those based on ZEUS-S in most of the investigated phase-space region. Especially at large $E_{T}^{\text {jet }}$, the usage of MSTW08 instead of ZEUS-S in the NLO QCD calculations leads to higher predictions. The high-precision measurements of inclusive-jet photoproduction have the potential to constrain the proton PDFs in future QCD fits. 

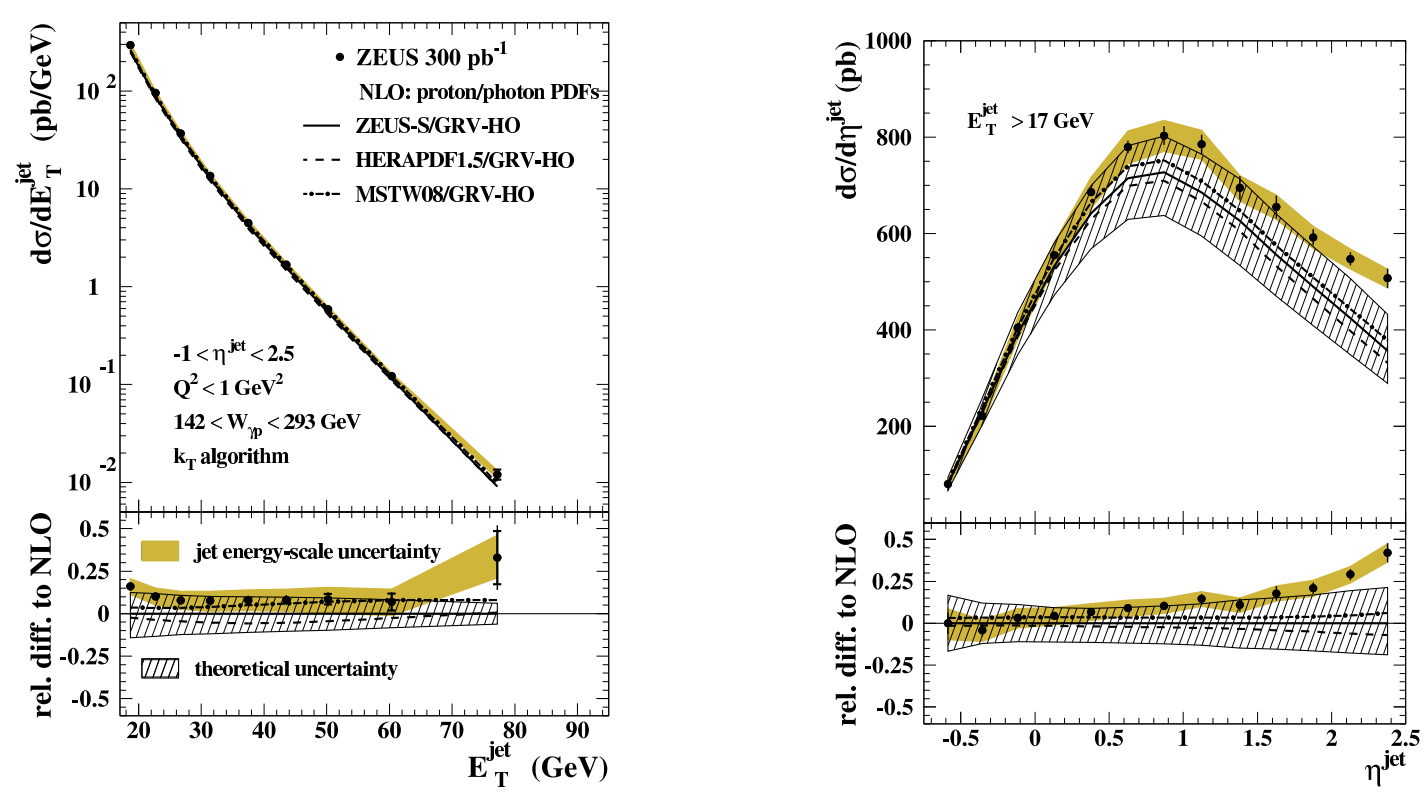

Figure 1: Single-differential cross sections $d \sigma / d E_{T}^{\text {jet }}$ (left) and $d \sigma / d \eta^{\text {jet }}$ (right) based on the $k_{T}$ algorithm. The data are compared to NLO QCD predictions based on different proton PDFs.

In addition, inclusive-jet cross sections based on the $k_{T}$ algorithm were determined as functions of $E_{T}^{\text {jet }}$ in different regions of $\eta^{\text {jet }}$. As observed for the single differential cross sections, the data are well described by NLO QCD except at $E_{T}^{\text {jet }}<21 \mathrm{GeV}$ for $\eta^{\text {jet }}>2$.

\section{Impact of multi-parton interactions}

The effect of multi-parton interactions is not included in the NLO QCD calculations described in Sec. 1. Instead, correction factors were obtained using Pythia-MI including multi-parton interactions with a minimum transverse momentum of the secondary scatter, $p_{T, \min }^{\mathrm{sec}}$, of $1,1.5$ and $2 \mathrm{GeV}$. Single-differential cross sections based on the $k_{T}$ algorithm as functions of $E_{T}^{\text {jet }}$ and $\eta^{\text {jet }}$ are compared to NLO QCD predictions where these correction factors have been applied are shown in Fig. 2. The inclusion of multi-parton interactions increase the predictions at low $E_{T}^{\text {jet }}$ and large $\eta^{\text {jet }}$. The best description of the data is observed for $p_{T, \min }^{\mathrm{sec}}=1.5 \mathrm{GeV}$.

\section{Comparison of different jet algorithms}

Differential cross sections for inclusive-jet photoproduction as functions of $E_{T}^{\text {jet }}$ and $\eta^{\text {jet }}$ were measured for the $k_{T}$, anti- $k_{T}$ and SIScone jet algorithms. The hadronisation corrections are largest for the SIScone algorithm while similar corrections were found for the $k_{T}$ and anti- $k_{T}$ algorithms. As shown for the $k_{T}$ algorithm above, the measurements based on anti- $k_{T}$ and SIScone are well described by NLO QCD except at large $\eta^{\text {jet }}$.

To compare the different jet algorithms in detail, the rations of the measured cross sections anti- $k_{T} / k_{T}$, SIScone $/ k_{T}$ and anti- $k_{T} /$ SIScone were determined and are shown in Fig. 3. The cross 

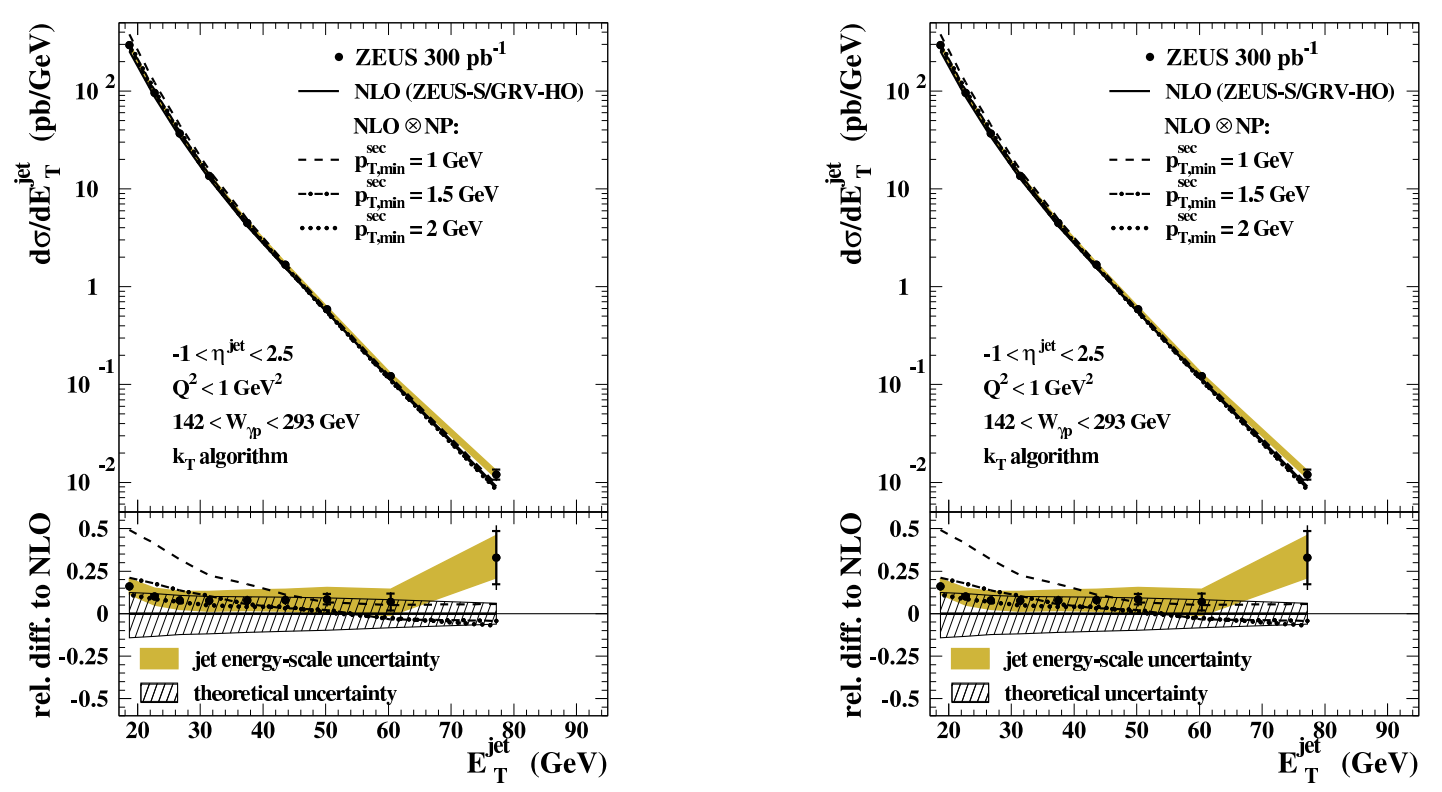

Figure 2: Single-differential cross sections $d \sigma / d E_{T}^{\text {jet }}$ (left) and $d \sigma / d \eta^{\text {jet }}$ (right) based on the $k_{T}$ algorithm. The data are compared to NLO QCD predictions. For comparison, the NLO QCD calculations including an estimation of non-perturbative effects are shown in addition.
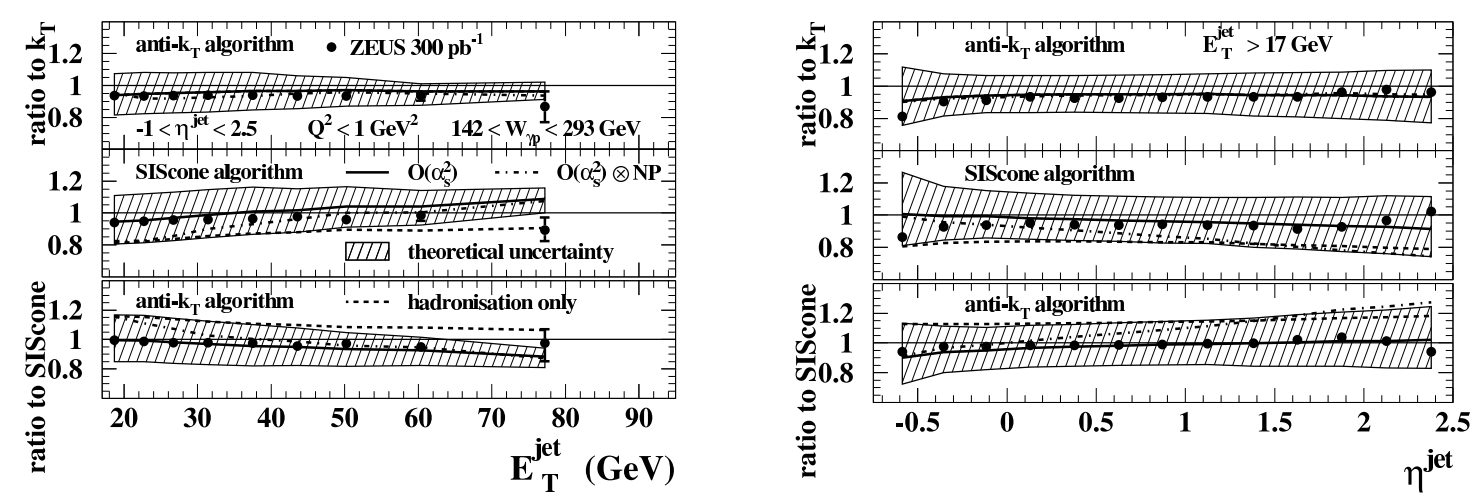

Figure 3: The ratios of the measured cross sections anti- $k_{T} / k_{T}$, SIScone $/ k_{T}$ and anti- $k_{T} / S I S c o n e$ as functions of $E_{T}^{\text {jet }}$ (left) and $\eta^{\text {jet }}$ (right).

sections for anti- $k_{T}$ have the same shape as those for $k_{T}$, but are about $6 \%$ smaller. The measured cross sections based on SIScone have a slightly different shape than those based on $k_{T}$ or anti- $k_{T}$. The QCD calculations with up to three partons in the final state describe the measured ratios.

\section{Determination of $\alpha_{s}$ and its energy-scale dependence}

The measured single-differential cross sections $d \sigma / d E_{T}^{\text {jet }}$ for $21<E_{T}^{\text {jet }}<71 \mathrm{GeV}$ based on the $k_{T}$, anti- $k_{T}$ and SIScone jet algorithms were used to determine $\alpha_{S}\left(M_{Z}\right)$ [14]. Consistent results were obtained for all three jet algorithms: 


$$
\begin{aligned}
\left.\alpha_{s}\left(M_{Z}\right)\right|_{k_{T}} & \left.=0.1206_{-0.0022}^{+0.0023} \text { (exp. }\right)_{-0.0035}^{+0.0042} \text { (th.), } \\
\left.\alpha_{S}\left(M_{Z}\right)\right|_{\text {anti- } k_{T}} & \left.=0.1198_{-0.0022}^{+0.0023} \text { (exp. }\right)_{-0.0034}^{+0.0041} \text { (th.), } \\
\left.\alpha_{s}\left(M_{Z}\right)\right|_{\text {SIScone }} & \left.=0.1196_{-0.0021}^{+0.0022} \text { (exp. }\right)_{-0.0043}^{+0.0046} \text { (th.). }
\end{aligned}
$$

The results are in agreement with other determinations of $\alpha_{S}\left(M_{Z}\right)$ [11]. In addition, values of $\alpha_{s}$ were extracted at the mean values, $\left\langle E_{T}^{\mathrm{jet}}\right\rangle$, of the bins in $E_{T}^{\mathrm{jet}}$ without assuming the running of $\alpha_{s}$. The extracted values of $\alpha_{s}$ as a function of $E_{T}^{\text {jet }}$ are shown in Fig. 4. This measurement confirms the running of $\alpha_{s}$ over a wide $E_{T}^{\text {jet }}$ range. The observed running is in good agreement with the two-loop QCD prediction.

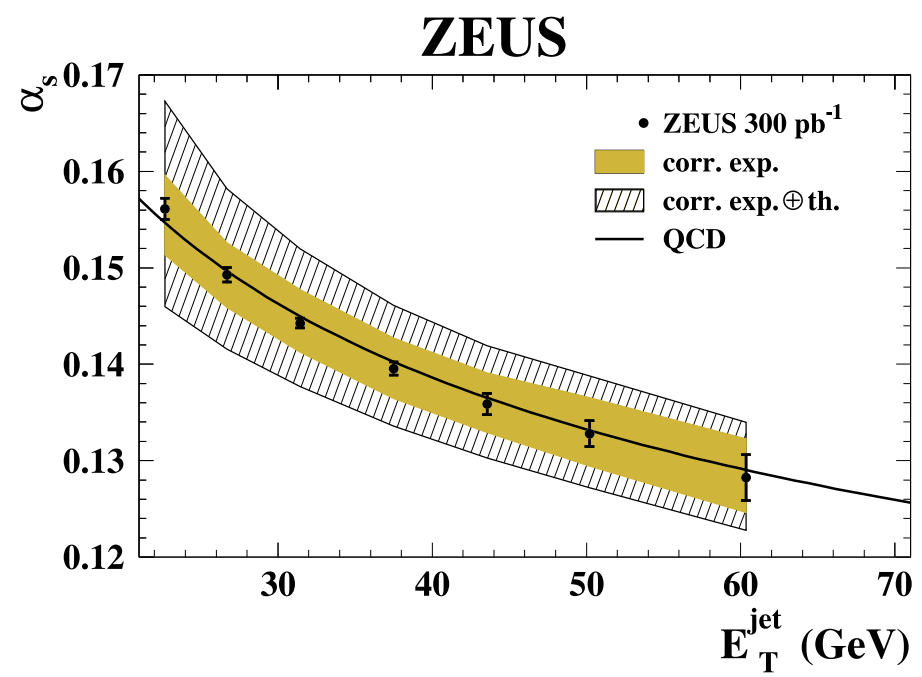

Figure 4: $\alpha_{s}$ extracted at various $\left\langle E_{T}^{\mathrm{jet}}\right\rangle$ values from the measured $d \sigma / d E_{T}^{\mathrm{jet}}$ cross sections based on the $k_{T}$ algorithm.

\section{Summary and conclusions}

Inclusive-jet cross sections in photoproduction were measured using the ZEUS detector. The data are generally well described by NLO QCD predictions. The inclusion of multi-parton interactions improves the predictions at low $E_{T}^{\text {jet }}$ and large $\eta^{\text {jet }}$. The presented measurements have the potential to improve the photon and proton PDFs in future QCD fits. The strong coupling constant was extracted at the $Z$ mass with competitive precision compared to other measurements and over a wide $E_{T}^{\text {jet }}$ range.

\section{References}

[1] S. Catani et al., Longitudinally-invariant $k_{\perp}$-clustering algorithms for hadron-hadron collisions, Nucl. Phys. B 406, 187 (1993).

[2] S.D. Ellis and D.E. Soper, Successive combination jet algorithm for hadron collisions, Phys. Rev. D 48, 3160 (1993). 
[3] M. Cacciari, G.P. Salam and G. Soyez, The anti- $k_{t}$ jet clustering algorithm, JHEP 04, 063 (2008).

[4] G.P. Salam and G. Soyez, A practical seedless infrared-safe cone jet algorithm, JHEP 05, 086 (2007).

[5] M. Klasen, T. Kleinwort and G. Kramer, Inclusive jet production in $\gamma p$ and $\gamma \gamma$ processes: direct and resolved photon cross sections in next-to-leading order QCD, Eur. Phys. J. C 1, 1 (1998).

[6] S. Chekanov et al., ZEUS next-to-leading-order QCD analysis of data on deep inelastic scattering, Phys. Rev. D 67, 012007 (2003).

[7] M. Glück, E. Reya, A. Vogt, Parton structure of the photon beyond the leading order, Phys. Rev. D 45, 3986 (1992);

M. Glück, E. Reya, A. Vogt, Photonic parton distributions, Phys. Rev. D 46, 1973 (1992).

[8] T. Sjöstrand, High-energy-physics event generation with PYTHIA 5.7 and JETSET 7.4, Comput. Phys. Comm. 82, 74 (1994).

[9] G. Marchesini et al., HERWIG 5.1 - a Monte Carlo event generator for simulating hadron emission reactions with interfering gluons, Comput. Phys. Comm. 67, 465 (1992);

G. Corcella et al., HERWIG 6: an event generator for hadron emission reactions with interfering gluons (including supersymmetric processes), JHEP 01, 010 (2001).

[10] T. Sjöstrand and M. van Zijl, A multiple-interaction model for the event structure in hadron collisions, Phys. Rev. D 36, 2019 (1987).

[11] H. Abramowicz et al., Inclusive-jet photoproduction at HERA and determination of $\alpha_{s}$, Nucl. Phys. B 864, 1 (2012) [arXiv: 1205 .6153].

[12] F.D. Aaron, Combined measurement and QCD analysis of the inclusive $e^{ \pm}$scattering cross sections at HERA, JHEP 01, 109 (2010).

[13] A.D. Martin et al., Parton distributions for the LHC, Eur. Phys. J. C 63, 189 (2009).

[14] S. Chekanov et al., Inclusive jet cross sections in the Breit frame in neutral current deep inelastic scattering at HERA and determination of $\alpha_{s}$, Phys. Lett. B 547, 164 (2002). 